\title{
La visión del estudiante respecto a la Diplomatura de Fisioterapia en la Universitat de València: un estudio descriptivo
}

\section{Gemma Victoria Espí López}

Profesora Colaboradora del Departamento de Fisioterapia de la

Universitat de València

gemma.espi@uv.es

\section{Inmaculada Colorado Lluch}

Profesora Asociada de Departamento de Estadística e Investigación

Operativa de la Universitat de València

m.inmaculada.colorado@uv.es

\section{María Jovani Palau}

Departamento de Estadística e Investigación Operativa de la

Universitat de València

maria.jovani@uv.es

\author{
José Sánchez Frutos \\ Profesor Titular del Departamento de Fisioterapia de la Universitat \\ de València \\ jose.sanchez-frutos@uv.es \\ Lirios Dueñas Moscardó \\ Profesora Colaboradora del Departamento de Fisioterpia de la \\ Universitat de València \\ lirios.duenas@uv.es

\section{Sofía Pérez Alenda} \\ Profesora Colaboradora del Departamento de Fisioterpia de la \\ Universitat de València \\ sofia.perez-alenda@uv.es
}

|Fecha presentación: 21/12/2011 | Aceptación: 02/07/2012 |Publicación: 20/12/2012

Resumen

Se ha llevado a cabo una encuesta con 116 alumnos de tercer curso de la diplomatura en junio de 2011 con diferentes aspectos de la titulación sobre inserción en el mundo profesional, satisfacción respecto a conocimientos teóricos y aplicaciones prácticas durante su formación, así como la calidad docente e infraestructura de la propia Universidad. El objetivo es afrontar en un futuro próximo estrategias de innovación metodológicas más adecuadas. El 63,8\% de los encuestados consideraron cumplidas las expectativas que les movieron a estudiar Fisioterapia y el $13 \%$ se sintieron poco preparados para iniciar la actividad laboral. El $100 \%$ de los encuestados consideraron que deberían seguir formándose, mostrando todos ellos interés en realizar un postgrado. Destaca el interés de profundizar en conocimientos sobre técnicas de terapia manual (81\%).

Palabras clave: educación, fisioterapia, satisfacción, calidad

\section{Resum}

S'ha dut a terme una enquesta amb 116 alumnes de tercer curs de la diplomatura en juny de 2011 amb aspectes de la titulació sobre inserció en l'àmbit professional, satisfacció respecte a coneixements teòrics i aplicacions pràctiques durant la seua formació, així com la qualitat docent e infraestructura de la pròpia Universitat. L'objectiu es afrontar en un futur pròxim estratègies d'innovació metodològiques més adequades. El 63,8\% dels enquestats consideraren complides les expectatives que els van moure a estudiar Fisioteràpia i el $13 \%$ es van sentir poc preparats per a iniciar l'activitat laboral. El 100\% dels enquestats consideraren que havien de seguir formant-se, mostrant tots ells interès en realitzar un postgrau. Destaca l'interés d'aprofundir en coneixements al voltant de tècniques de teràpia manual (81\%).

Paraules clau: educació, fisioteràpia, satisfacció, qualitat

\begin{abstract}
A survey was carried out among third-year with 116 students of the Diplomatura (old three-year degree), in June 2011 about different aspects of the degree of integration into the professional world, satisfaction with theoretical knowledge and practical applications for its training and teaching quality and infrastructure of the University. The objective is to face, most appropriate innovation methodological strategies in the near future. $63.8 \%$ of participants think that the expectations which led them to study physical therapy have been met and $13 \%$ do not feel very ready to start professional activity. $100 \%$ of participants think they should continue education, and $100 \%$ of subjects showed interest in following a postgraduate program. Destaca el interés de profundizar en conocimientos sobre técnicas de terapia manual (81\%).
\end{abstract}

Key words: education, physical therapy, satisfaction, quality 


\section{Introducción}

La Escuela Universitaria de Fisioterapia de Valencia, actualmente Facultad, fue la primera en incorporar la Diplomatura de Fisioterapia en un Plan Docente, comenzando a impartir sus clases en el curso académico 1983-1984. Como consecuencia de los acuerdos adoptados en la Declaración de Bolonia (MECD, 2003; Bologna Process Web Site, 2005) en 1999 para la creación de un Espacio Europeo de Educación Superior, las Universidades españolas, entre ellas la de Valencia, están siendo pioneros de importantes transformaciones en la estructura de sus estudios. En el año 2004, la Agencia Nacional de Evaluación de la Calidad y Acreditación (ANECA) publicó el Libro Blanco de Título de grado en Fisioterapia (ANECA, 2004; Rebollo, 2008; Universitat de Valencia, 2011) en el que se propuso un Título de grado de 240 créditos que comprendiera cuatro años, estableciéndose las competencias transversales y específicas de los fisioterapeutas, los objetivos y la estructura general del título. Así, en la Universitat de València entraron en vigor las nuevas titulaciones de grado en el curso 2009-2010, conviviendo con la diplomatura hasta el pasado curso 2010-2011, coexistiendo con los nuevos elementos metodológicos(De Miguel, 2004; Jiménez, 2005).

El plan de estudios de la diplomatura de Fisioterapia tiene sus antecedentes en la Ley de Reforma Universitaria (Ley Orgánica 11/83 de 25 de agosto, de Reforma Universitaria. BOE $\mathrm{n}^{0}$ 209, 01/09/1983). Dentro de éste plan se incluían una serie de asignaturas en las que las clases prácticas asumían un rol importante y que contribuyeron enormemente a formar fisioterapeutas así como su destreza en la aplicación de las técnicas fisioterápicas. Éste es el caso de la asignatura Tratamiento Fisioterápico en las Lesiones del Sistema Nervioso Periférico y Enfermedades Musculares que se impartió por última vez en el curso académico 2010-2011, considerada en estrecha relación con el resto de materias que componían el curriculum de la diplomatura de Fisioterapia. Actualmente en el grado los contenidos de esta asignatura se incluyen en el curriculum grado pero diseminados y generalizados en diferentes materias como son: Métodos específicos de intervención en fisioterapia y Fisioterapia en Especialidades Clínicas (Facultat de Fisioteràpia, 2011).

En este estudio (GADE, 2004) nos proponemos detectar algunos puntos fuertes y débiles en la diplomatura no contenidos en el Informe final de la Diplomatura de Fisioterapia en el 2004 y compararlos con los recientemente implantados estudios de grado y postgrado, así como valorar la visión del estudiante respecto diferentes metodologías de cara a plantear nuevas estrategias de innovación metodológicas.

Por ello, la elaboración de este estudio pretende conocer, mediante la modalidad de encuesta, la visión y satisfacción del estudiante respecto a diferentes aspectos de la titulación: inserción en el mundo profesional; satisfacción respecto a conocimientos teóricos y aplicaciones prácticas durante su formación, así como la calidad docente y de infraestructura de la propia Universidad. Específicamente preguntamos sobre la asignatura Tratamiento Fisioterápico en las Lesiones del

\begin{tabular}{|c|c|}
\hline \multirow{10}{*}{ General fisioterapia } & ¿Has cumplido las expectativas que te movieron a estudiar Fisioterapia? \\
\hline & ¿Piensas que estás preparado para iniciar la actividad profesional como Fisioterapeuta? \\
\hline & ¿Piensas que todavía deberías formarte realizando cursos específicos para Fisioterapeutas? \\
\hline & $\begin{array}{l}\text { ¿Piensas que tu incorporación profesional por cuenta propia o ajena va a ser de forma in- } \\
\text { mediata? }\end{array}$ \\
\hline & $\begin{array}{l}\text { ¿De todas las asignaturas de Fisioterapia, en cuál o cuáles te gustaría haber profundizado?. } \\
\text { Enuméralas }\end{array}$ \\
\hline & $\begin{array}{l}\text { ¿Piensas que en muchos temas convendría realizar las prácticas antes que los contenidos } \\
\text { teóricos? }\end{array}$ \\
\hline & $\begin{array}{l}\text { ¿Cómo integras mejor las técnicas fisioterápicas? } \\
\text { Con vídeos Por medio de fotografías Por mis apuntes Por mi propia búsqueda en } \\
\text { libros o internet }\end{array}$ \\
\hline & $\begin{array}{l}\text { ¿Te ha ayudado todo el material que te ha proporcionado el profesorado a través del aula } \\
\text { virtual? }\end{array}$ \\
\hline & ¿Te han resultado provechosas las prácticas hospitalarias? \\
\hline & ¿Te han resultado provechosas las prácticas en centros privados? \\
\hline \multirow{2}{*}{$\begin{array}{l}\text { Tratamiento Fisioterápico en } \\
\text { las Lesiones del Sistema } \\
\text { Nervioso Periférico y } \\
\text { Enfermedades Musculares }\end{array}$} & ¿Te ha parecido provechosa la asignatura de SNP? \\
\hline & $\begin{array}{l}\text { De los tipos de técnicas que se han visto en la práctica, ċen cuál o cuáles te gustaría profundizar? } \\
\text { Masaje Terapia Manual Miofascial Neuromeníngeo }\end{array}$ \\
\hline \multirow[b]{2}{*}{ Formación postgrado } & ¿Te gustaría seguir tus estudios realizando un postgrado? \\
\hline & $\begin{array}{l}\text { ¿Sobre qué temas te gustaría realizarlo? } \\
\text { Fisioterapia Deportiva } \quad \text { Fisioterapia infantil } \quad \text { Terapia Manual } \quad \text { Fisioterapia Geriátrica }\end{array}$ \\
\hline Calidad docente & ¿En general, qué te ha parecido la calidad docente? \\
\hline Adecuación espacios docentes & ¿En general, qué te ha parecido la estructura, material y aulas? \\
\hline
\end{tabular}

Tabla 1. Encuesta con las dimensiones y preguntas que se incluyen. 
Sistema Nervioso Periférico y Enfermedades Musculares, ya que consideramos que era un punto fuerte de la titulación y al haber sido diseminados sus contenidos puede haberse convertido en una debilidad. Las preguntas respecto a las perspectivas formativas de postgrado van orientadas a valorar la motivación para realizar los actuales másteres oficiales y así como abrir posibilidades a plantear nuevas opciones.

\section{Material y método}

Se ha llevado a cabo una encuesta en junio de 2011 a los alumnos de tercer curso de la diplomatura de Fisioterapia en la Universidad de Valencia al finalizar la asignatura cuatrimestral obligatoria Tratamiento Fisioterápico en las Lesiones del Sistema Nervioso Periférico y Enfermedades Musculares.

Esta encuesta incluye 16 ítems clasificados en diferentes dimensiones como son: generalidades sobre fisioterapia; la asignatura Tratamiento Fisioterápico en las Lesiones del Sistema Nervioso Periférico y Enfermedades Musculares; formación postgrado en fisioterapia; calidad docente; y adecuación de los espacios docentes (Tabla 1). Para la mayoría de las respuestas se utilizó una escala Likert de 4 puntos con las opciones algo, poco, bastante o mucho. Se incluyeron 3 preguntas de opción múltiple y una abierta.

Análisis estadístico

Tras recoger las encuestas, se procedió a la codificación y análisis de datos mediante los programas informáticos SPSS v19 y Microsoft Excel 2010. En primer lugar se realizó el análisis de independencia de las variables respecto a edad y sexo. Posteriormente se realizó el análisis descriptivo de la muestra y de las respuestas. Las variables categóricas se han descrito mediante sus frecuencias, medias y desviaciones típicas. Para contrastar la posible relación entre algunas variables se utilizó la prueba exacta de Fisher.

La comparación de medias se realizó mediante ANOVA. En caso de normalidad pero de incumplirse la homogeneidad de varianzas se utilizó la prueba robusta de igualdad de medias de Welch. Para el análisis post-hoc, se utilizaron las pruebas que no sumasen homogeneidad de varianzas: Tamhane, Dunnett y Games-Howel. El nivel de significación utilizado ha sido 0,05

\section{Resultados}

Han formado parte del estudio 116 sujetos de los cuales 47 son varones $(40,5 \%)$ y 69 mujeres $(59,5 \%)$. El 75,7\% de la muestra tienen entre 18 y 25 años; el 21,7\% entre 26 y 35; y el 2,6\% más de 35 años. Después de analizar por grupos los distintos ítems, no se apreciaron diferencias significativas ni por sexo, ni por edad, por lo que, el análisis final se realizó sin utilizar estos factores, considerando todos los datos como una única muestra. A continuación comentamos los resultados obtenidos, resumidos en la Tabla 2.

El 63,8\% de los encuestados consideraron que se han cumplido bastante o mucho las expectativas que les motivaron a estudiar Fisioterapia. Sobre la percepción de la preparación del encuestado para iniciar la actividad profesional, los resultados muestran que el $13 \%$ de los encuestados se sienten poco preparados. Al analizar los ítems sobre las ex-

\begin{tabular}{|c|c|c|c|c|c|}
\hline Cuestiones & $\begin{array}{l}\text { Poco } \\
\text { frec abs } \\
(\%)\end{array}$ & $\begin{array}{l}\text { Algo } \\
\text { frec abs } \\
(\%)\end{array}$ & $\begin{array}{l}\text { Bastante } \\
\text { frec abs } \\
(\%)\end{array}$ & $\begin{array}{l}\text { Mucho } \\
\text { frec abs } \\
(\%)\end{array}$ & $\begin{array}{c}\text { Media } \\
\text { (DT) }\end{array}$ \\
\hline $\begin{array}{l}\text { ¿Has cumplido las expectativas que te movieron a estu- } \\
\text { diar fisioterapia? }\end{array}$ & $11(9,5)$ & $31(26,7)$ & $55(47,4)$ & $19(16,4)$ & $\begin{array}{c}2,71 \\
(0,85)\end{array}$ \\
\hline $\begin{array}{l}\text { ¿Piensas que estás preparado para iniciar la actividad } \\
\text { profesional como Fisioterapeuta? }\end{array}$ & $15(13,0)$ & $50(43,5)$ & $42(36,5)$ & $8(7,0)$ & $\begin{array}{c}2,37 \\
(0,80)\end{array}$ \\
\hline $\begin{array}{l}\text { ¿Piensas que todavía deberías formarte realizando cur- } \\
\text { sos específicos para Fisioterapeutas? }\end{array}$ & $\mathrm{o}(0,0)$ & $3(2,6)$ & $14(12,2)$ & $98(85,2)$ & $\begin{array}{c}3,83 \\
(0,44)\end{array}$ \\
\hline $\begin{array}{l}\text { ¿Piensas que tu incorporación profesional por cuenta } \\
\text { propia o ajena va a ser de forma inmediata? }\end{array}$ & $31(27,7)$ & $51(45,5)$ & $28(25,0)$ & $2(1,8)$ & $\begin{array}{c}2,01 \\
(0,78)\end{array}$ \\
\hline ¿Te ha parecido provechosa la asignatura de SNP? & $1(0,9)$ & $19(16,5)$ & $70(60,9)$ & $25(21,7)$ & $\begin{array}{c}3,03 \\
(0,65)\end{array}$ \\
\hline $\begin{array}{l}\text { ¿Piensas que en muchos temas convendría realizar las } \\
\text { prácticas antes que los contenidos teóricos? }\end{array}$ & $29(25,7)$ & $42(37,2)$ & $28(24,8)$ & $14(12,4)$ & $\begin{array}{c}2,24 \\
(0,97)\end{array}$ \\
\hline ¿Te han resultado provechosas las prácticas hospitalarias? & $9(8,0)$ & $33(29,2)$ & $42(37,2)$ & $29(25,7)$ & $\begin{array}{c}2,81 \\
(0,91)\end{array}$ \\
\hline $\begin{array}{l}\text { ¿Te han resultado provechosas las prácticas en centros } \\
\text { privados? }\end{array}$ & $6(5,4)$ & $14(12,6)$ & $31(27,9)$ & $60(54,1)$ & $\begin{array}{c}3,31 \\
(0,89)\end{array}$ \\
\hline $\begin{array}{l}\text { ¿Te gustaría seguir tus estudios realizando un pos- } \\
\text { tgrado? }\end{array}$ & $\mathrm{o}(0,0)$ & $13(11,2)$ & $52(44,8)$ & $51(44,0)$ & $\begin{array}{c}3,33 \\
(0,67)\end{array}$ \\
\hline En general, ¿̇qué te ha parecido la calidad docente? & $4(3,5)$ & $26(22,6)$ & $69(60,0)$ & $16(13,9)$ & $\begin{array}{c}2,84 \\
(0,70)\end{array}$ \\
\hline $\begin{array}{l}\text { En general, ¿̇qué te ha parecido la estructura, material y } \\
\text { aulas? }\end{array}$ & $10(8,6)$ & $42(36,2)$ & $54(46,6)$ & $10(8,6)$ & $\begin{array}{l}2,55 \\
(0,77)\end{array}$ \\
\hline
\end{tabular}

Tabla 2. Resultados de los ítems sobre la diplomatura que incluyen como posibles respuestas: poco, algo, bastante y mucho. Frec abs. Frecuencias absolutas; \%. Frecuencias relativas; DT. Desviación típica. 


\begin{tabular}{|l|l|}
\hline Asignatura & frec abs (\%) \\
\hline Fisioterapia del Aparato Locomotor & $66(65,35 \%)$ \\
\hline $\begin{array}{l}\text { Sistema Nervioso Central y Sistema } \\
\text { Nervioso Periférico }\end{array}$ & $54(53,47 \%)$ \\
\hline Anatomía y Biomecánica & $29(28,71 \%)$ \\
\hline Diagnóstico fisioterápico & $16(15,84 \%)$ \\
\hline Fisioterapia deportiva & $10(9,90 \%)$ \\
\hline Fisioterapia respiratoria y cardiovascular. & $5(4,95 \%)$ \\
\hline Electroterapia & $4(3,96 \%)$ \\
\hline Nuevos métodos de Terapia Manual & $3(2,97 \%)$ \\
\hline
\end{tabular}

Tabla 3. Resultados de las materias en las que el estudiante hubiera profundizado. Frec abs. Frecuencias absolutas; \%. Frecuencias relativas

pectativas y la preparación observamos que existe relación entre ellos (Fisher, $\mathrm{p}=\mathbf{0}, 000$ ). Concretamente, a mayor puntuación en las expectativas, mayor puntuación en estar preparado para iniciar la actividad profesional.

Respecto a la conveniencia de seguir realizando formación específica, los resultados mostraron que el 100\% de los encuestados piensan que deberían seguir formándose, destacando el $85,2 \%$ que eligieron la respuesta mucho. La percepción de estar preparado para iniciar la actividad profesional fue independiente de la percepción de necesitar formación de cursos específicos, la gran mayoría piensa que necesita más formación específica (independientemente de que se sientan preparados) (Fisher, $\mathrm{p}=0,10$ ).

Sobre las técnicas que les gustaría profundizar vistas en la práctica, el 45,7\% eligió una sola opción; el 33,6\% eligió 2 opciones; el 12,1\% eligió 3 opciones; sólo un 7,8\% eligió todas. Sólo hay un individuo que no eligió ninguna. Las opciones marcadas según la preferencia son Terapia Manual (81,0\%), Movilización neuromeníngea (39,7\%), Técnica Miofascial (39,7\%) y Masaje (19,8\%). Si observamos los que han contestado solo una opción, de ellos, el 71,7\% eligieron Terapia Manual.

El 73,2\% de los encuestados piensan que su incorporación profesional no será de forma inmediata ( росо y algo). Sobre las asignaturas de Fisioterapia en las que les gustaría haber profundizado, que se le planteó al alumno como pregunta abierta, destacando el alto porcentaje que desearía haberlo hecho en Fisioterapia del Aparato Locomotor y en Sistema Nervioso Central y Periférico como se muestra en la Tabla 3

En la pregunta específica respecto si les pareció provechosa la asignatura Tratamiento Fisioterápico en las Lesiones del Sistema Nervioso Periférico y Enfermedades Musculares, al 82,6\% de los sujetos les pareció bastante o muy provechosa. Respecto a las prácticas realizadas, al 25,7\% de los encuestados les resultaron muy provechosas las prácticas asistenciales hospitalarias, y en centros privados al 54,1\%.

Respecto a las preferencias en metodología docente, el $64,7 \%$ de los sujetos eligió una sola opción, el 27,6\% eligió 2 opciones, el 6\% eligió 3 opciones, nadie eligió todas. 2 individuos no eligieron ninguna. Las opciones preferidas son: vídeos (92,2\%), apuntes (26,7\%), fotografías (12,9\%), o la búsqueda de información (6\%). Si observamos los que han contestado solo una opción, el 93,33\% de ellos, prefirieron vídeos.

Respecto a la pregunta sobre la ayuda del material proporcionado por el profesor en el aula virtual, en la que se opta por tres posibles respuestas (nada, en parte y mucho) el 74,5\% piensan que les ha ayudado el material proporcionado en el aula virtual, sólo un 20,2\% y un 5,3\% opinaron que en parte o nada (media $=1,31 ; \mathrm{DT}=0,56$ ).

Los temas sobre los que les gustaría profundizar mediante postgrado fueron: en primer lugar Terapia manual (62,93\%), seguido de Fisioterapia Deportiva (39,66\%) e Infantil $(35,21 \%)$ que mostraron resultados similares y, por último, Geriatría con menor interés (10,34\%). Destaca el interés de realizar un postgrado en diferentes materias en el $100 \%$ de los sujetos.

En la pregunta sobre la calidad docente, el 73,9\% consideraron que la docencia tuvo bastante o mucha calidad. En cuanto al material y las aulas, el 82,8\% de los sujetos estaban algo o bastante satisfechos, y el 8,6\% poco o mucho en la misma proporción.

\section{Discusión}

Los estudiantes de la diplomatura han visto cumplidas sus expectativas al cursar los estudios. Casi a la mitad de los estudiantes no les pareció estar muy preparados a la hora de comenzar su andadura profesional, ni pensaban que su incorporación al mundo laboral fuera de forma inmediata mostrándose algo confusos con su futuro en este sentido, por lo que piensan que necesitan continuar con su formación, mostrando gran interés por ampliar conocimientos una vez concluida su diplomatura en cursos de postgrado. De hecho, cuando les preguntamos sobre qué materias realizarían un postgrado de entre las cuatro que hemos considerado como las más representativas, mostraron preferencia por la Terapia manual, siguiéndole con bastante diferencia la Fisioterapia Deportiva y Fisioterapia Infantil con puntuaciones similares, y poco por la Fisioterapia Geriátrica. En este sentido, en el grado ya se incluyen estos conocimientos en la materia Fisioterapia en Especialidades Clínicas. Un punto muy positivo de este estudio fue que las preferencias de las materias que eligieron son muy dinámicas y los alumnos muestran interés en profundizar en todas ellas ya que piensan que les puede llevar a aplicar los futuros tratamientos con mayor seguridad. Si bien los docentes hemos de mostrarles la diversidad existente en las diferentes especialidades y métodos mediante los másteres que ofrece actualmente la Facultat, así como otras opciones ofertadas por otras Universidades.

De las materias impartidas en la diplomatura, cabe destacar el interés por profundizar en materias sobre Fisioterapia del Aparato Locomotor, Fisioterapia del Sistema Nervioso Central y Periférico con una gran diferencia respecto a las demás. Es importante resaltar cómo el estudiante se interesa por los conocimientos generales de anatomía y biomecánica, necesarias para desempeñar la anterior. Ya con mayor diferencia les interesa el diagnóstico fisioterápico y las demás especialidades de Fisioterapia. Estos resultados pueden ser un indicativo de que quizás las horas que se dedican a cada una de ellas sean insuficientes o bien que requieran mayor profundidad. Debemos tener en cuenta la diversidad e importante cantidad de conocimientos de materias generales y específicas que se incluyen en el curriculum de fisioterapia. Por ello, y en relación con otras respuestas, pensamos que la mayoría de los sujetos (98 de 116) perciben la terapia manual como muy provechosa, aunque ello implique alcanzar importantes conocimientos anatómicos y biomecánicos. Le sigue de cerca la preferencia en técnicas miofasciales y de movilización neuromeníngea, probablemente por tratarse de técnicas de aplicación menos intensas y que requieren mayor concentración. Sorprendentemente por el masaje muestran un interés menor. En este punto los docentes deberíamos hacer un es- 
fuerzo por recuperar en ellos el entusiasmo de la aplicación de una técnica que se ha demostrado tan eficaz en la recuperación de lesiones del aparato locomotor.

No parece motivarles mucho realizar las prácticas antes de la teoría, sin embargo en algunas materias puede resultar muy interesante para asentar mejor los conocimientos. Este punto podría servir como punto de partida en futuras investigaciones.

Las nuevas metodologías, y concretamente las Nuevas Tecnologías de la Información y de la Comunicación (NTIC) abren innumerables posibilidades al conocimiento. En el caso de la integración de conocimientos a través de éstas técnicas, los estudiantes ven muy positivas las reproducciones de las técnicas mediante vídeos que les aproxima de una forma real a las mismas. También para ellos los apuntes tomados en clase constituyen un pilar importante, sin embargo no muestran mucho interés por realizar búsquedas de información en libros o internet, probablemente por el trabajo añadido que supone y por el alto coste económico de los libros sobre la materia. Actualmente, a través del aula virtual se les facilita abundante información, sin embargo se debería potenciar la búsqueda autónoma de información para completar los conocimientos. Llama la atención el poco interés por la búsqueda en internet de información complementaria de la materia, sin embargo con el grado se ha implantado una nueva asignatura (NTIC) que guiará al alumno a optimizar la búsqueda de información en este sentido.

En el apartado de las prácticas asistenciales en centros públicos y privados, cabe destacar la evidente valoración positiva respecto a su aprovechamiento, éstos últimos con mayor preferencia, probablemente por verse más identificados con sus posibilidades de acceso profesional, además de recibir un trato más individualizado.

Finalmente, podemos destacar que la mayoría de los estudiantes consideran que existe una buena calidad docente en la diplomatura, así como de las infraestructuras que ofrece la Facultad de Fisioterapia.

Las instituciones universitarias han de adaptar los procesos de enseñanza-aprendizaje con el objetivo de acentuar la implicación activa del estudiante en su proceso de aprendizaje, dando énfasis a la adquisición tanto de conocimientos como de habilidades, preparando al estudiante para asumir responsabilidades en un mundo en rápido y constante cambio, mejorando sus capacidades emocionales e intelectuales y dotándoles de las herramientas necesarias para que puedan enfrentarse a las exigencias de un procedimiento de formación continuada a lo largo de su vida. En este contexto, los métodos tradicionales siguen siendo necesarios, pero no suficientes para conseguir estos objetivos. Es indiscutible la necesidad de introducir procesos de innovación docente para mejorar el aprendizaje. Cabe recordar que sobre la base de los pilares básicos de la educación de Jacques Delors (1996), se añade la capacidad de aprender a pensar, fomentándose de esta manera la innovación.

\section{Conclusiones}

Los estudiantes están satisfechos con la diplomatura, pero muestran inseguridad en la incorporación inmediata del mundo laboral y pretenden continuar su formación en las diferentes materias basadas en las técnicas fisioterápicas de terapia manual. Se sienten atraídos por profundizar en las nuevas tendencias de tratamiento y poco por las tradicionales como el masaje. Por otro lado, ven positivas las prácticas hospitalarias y en centros privados, viéndose el gran esfuerzo realizado por los coordinadores de que organizan esta materia. También es necesario dar a conocer la importancia de los tratamientos en el sector de los procesos y tratamientos en el envejecimiento, así como de otros cursos de postgrado que se ofertan tanto en la Universitat de València como en otras universidades para responder las inquietudes profesionales y académicas del estudiante.

\section{Bibliografía}

Agencia Nacional de Evaluación de la Calidad y Acreditación (2004). Libro blanco de la Titulación de Fisioterapia Madrid: ANECA.

http://www.aneca.es/var/media/150428/libroblanco_juno5_fisioterapia.pdf

Bologna Process Web Site (2005). From Berlin to Bergen and beyond. http://www.bologna-bergen2005.no

De Miguel, Mario (2004). Modalidades de enseñanza centradas en el desarrollo de competencias. Orientaciones para promover el cambio metodológico en el Espacio Europeo de Educación Superior. Oviedo: Universidad de Oviedo.

Delors, Jacques (1996). La educación encierra un tesoro. Informe a la UNESCO de la Comisión Internacional sobre la educación para el siglo XXI. Madrid: Santillana. Ediciones UNESCO.

Facultat de Fisioteràpia (2011). Plan de estudios de la Facultat de Fisioteràpia. Universitat de València. http://www.uv.es/uvweb/fisioterapia/ca/estudisgrau/graus/oferta-graus/graus-1285847815795.html

Gabinet d'Avaluació i Diagnòstic Educatiu de la Universitat de València (2004). Informe final de la Diplomatura de Fisioterapia de la Escuela Universitaria de Fisioterapia. II Plan de Calidad de Universidades.

Jiménez, $\mathrm{M}^{\mathrm{a}}$ del Rocío (2005). Aplicación del crédito europeo en las aulas universitarias. En: Colás P, De Pablos J, coordinadores. La Universidad en la Unión Europea: el Espacio Europeo de Educación Superior y su impacto en la docencia. Archidona: Aljibe, pp.155-86.

M.E.C.D. (2003). La Integración del Sistema Universitario Español en el Espacio Europeo de Enseñanza Superior, Documento-Marco febrero de 2003. Madrid: Ministerio de Educación, Cultura y Deporte. http://www.unizar.es/eees/doc/Doc_Ministerio_12febo3.pdf

Rebollo, Jesús (2008). Contundente apoyo legislativo de las competencias profesionales enunciadas en el Libro Blanco de Fisioterapia. Fisioterapia, 30(1): pp. 1-4. http://dx.doi.org/10.1016/So211-5638(08)72949-3.

Universitat de Valencia (2011). Verifica del Título de Grado en Fisioterapia; http://www.uv.es/graus/qualitat/MV/C/1202_MV.pdf 